\title{
Selected Papers from the International Conference on Information, Communication, and Engineering 2013
}

\author{
Teen-Hang Meen, ${ }^{1}$ Stephen D. Prior, ${ }^{2}$ Kuei-Shu Hsu, ${ }^{3}$ and Artde Donald Kin-Tak Lam ${ }^{4}$ \\ ${ }^{1}$ Department of Electronic Engineering, National Formosa University, Yunlin 632, Taiwan \\ ${ }^{2}$ Department of Aeronautics, Astronautics and Engineering, University of Southampton, \\ Southampton SO17 1BJ, UK \\ ${ }^{3}$ Department of Applied Geoinformatics, Chia Nan University of Pharmacy \& Science, \\ Tainan City 717, Taiwan \\ ${ }^{4}$ College of Physics and Information Engineering, Fuzhou University, Fuzhou, Fujian 350116, China \\ Correspondence should be addressed to Teen-Hang Meen; thmeen@nfu.edu.tw
}

Received 6 April 2014; Accepted 6 April 2014; Published 8 May 2014

Copyright (C) 2014 Teen-Hang Meen et al. This is an open access article distributed under the Creative Commons Attribution License, which permits unrestricted use, distribution, and reproduction in any medium, provided the original work is properly cited.

2013 International Conference on Information, Communication and Engineering (ICICE 2013) is organized by China University of Petroleum (Huadong, East China) and Taiwanese Institute of Knowledge Innovation and held in Qingdao, Shandong, People's Republic of China on October 26-November 1, 2013. ICICE 2013 has received 653 submitted papers from 10 countries, whereby 214 papers have been selected by the committees to be presented in the ICICE 2013. The conference provides a unified communication platform for researchers in a wide area of topics from information technology, communication science, applied mathematics, computer science, advanced material science, and engineering. ICICE 2013 enables interdisciplinary collaboration between science and engineering technologists in the academic and industrial fields as well as networking internationally.

Mechanical engineering and design innovations are both an academic and practical engineering field that involves systematic technological materialization through scientific principles and engineering designs. Technological innovation by mechanical engineering includes IT-based intelligent mechanical systems, mechanics, and design Innovations. ITbased intelligent mechanical systems, which implant intelligence to machine systems, are an interdisciplinary area combining conventional mechanical technology and new information technology. The main goal of IT-based intelligent mechanical systems is to develop fundamental technologies and to apply them to the industry field as follows:

(i) computer-based design;

(ii) intelligent manufacturing system;

(iii) virtual manufacturing system;

(iv) core parts and devices for intelligent systems;

(v) computational engineering science;

(vi) design methodology and optimization;

(vii) machine diagnostics \& reliability;

(viii) human-machine interaction/virtual reality and entertainment.

This special issue selects 47 papers from ICICE 2013. This special issue enables interdisciplinary collaboration between material science and engineering technologists in the academic and industrial fields.

Teen-Hang Meen Stephen D. Prior

Kuei-Shu Hsu Artde Donald Kin-Tak Lam 


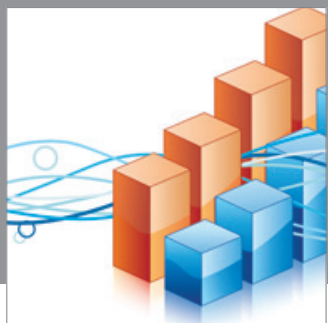

Advances in

Operations Research

mansans

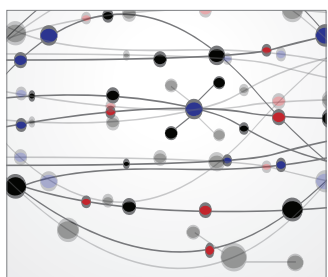

The Scientific World Journal
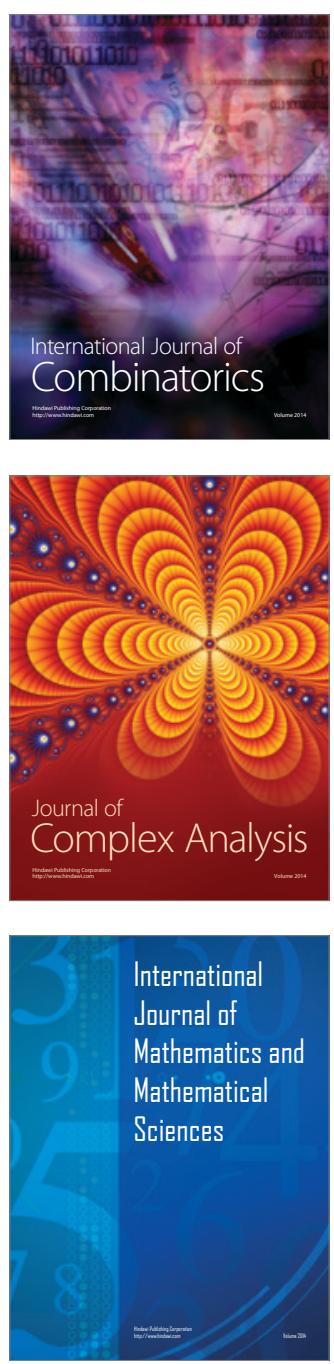
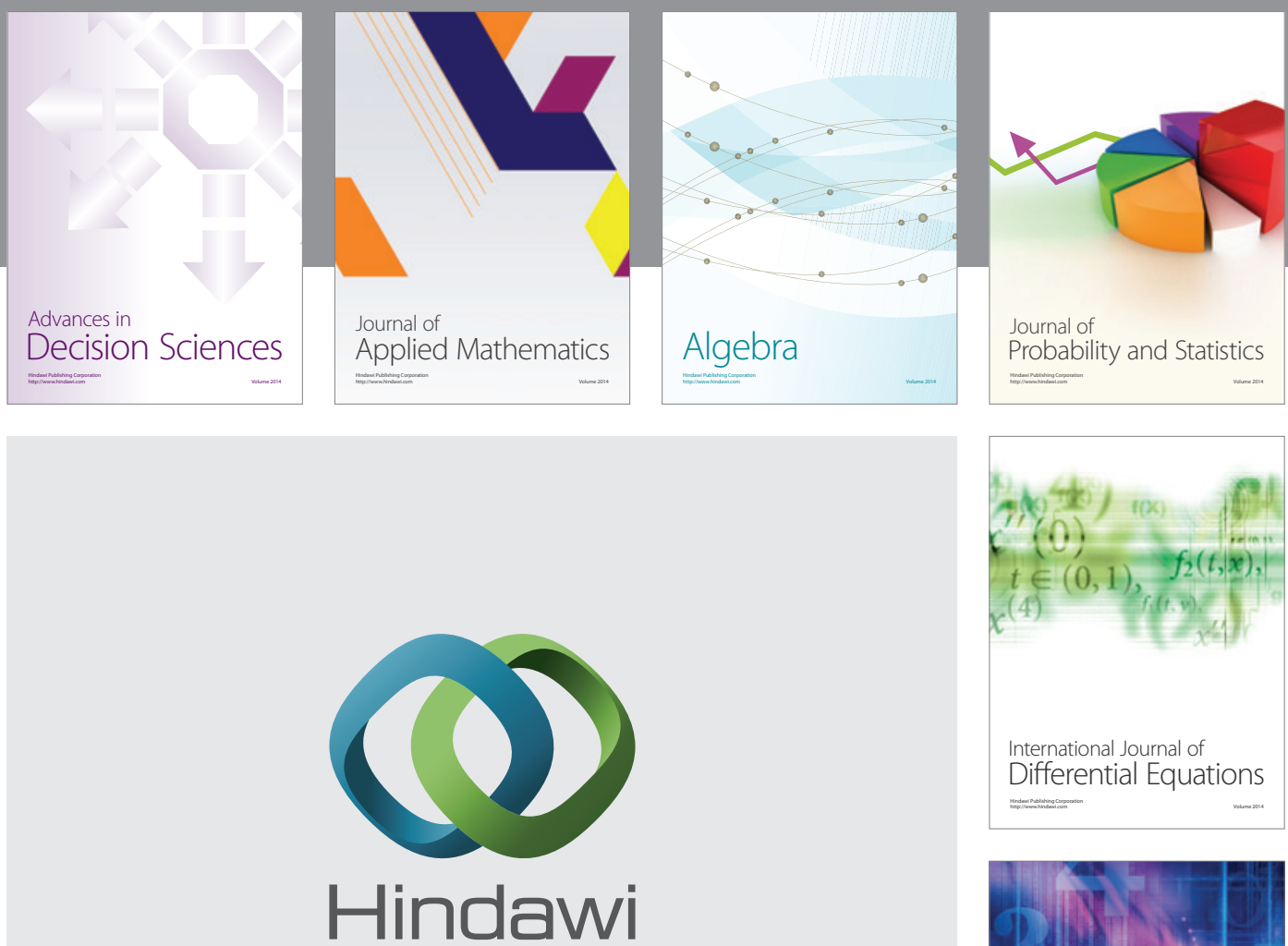

Submit your manuscripts at http://www.hindawi.com
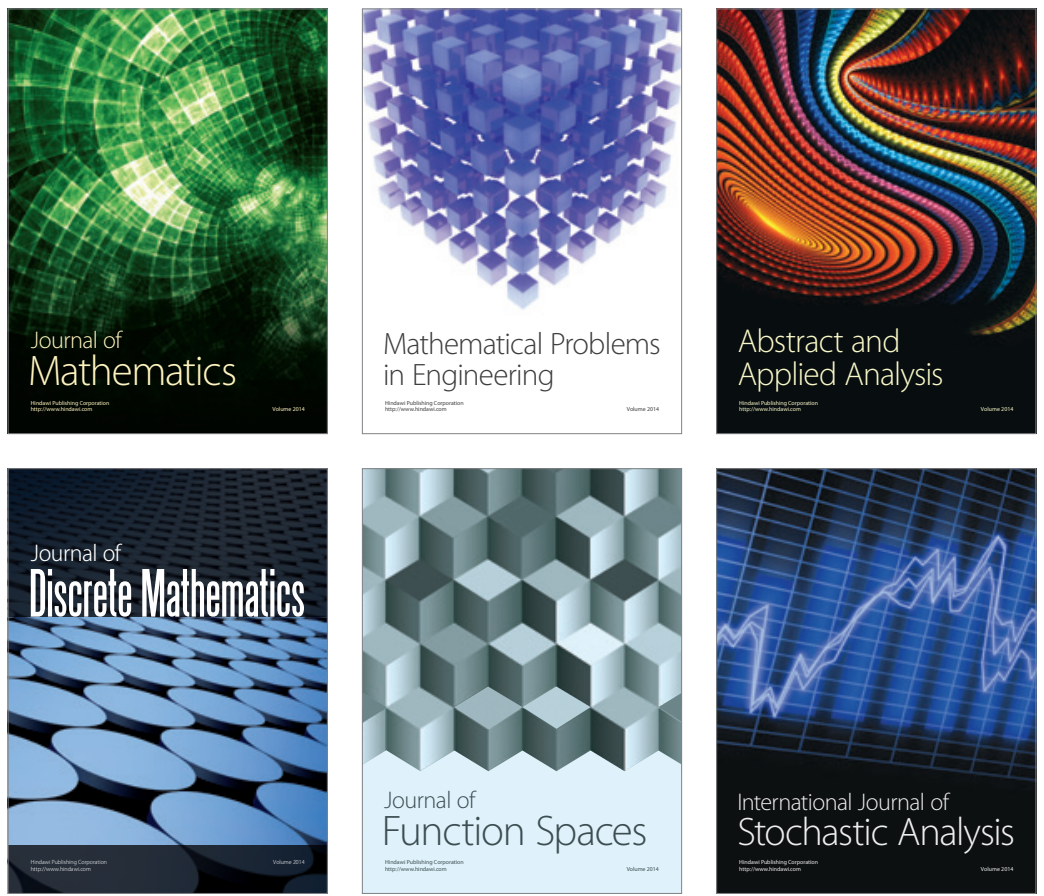

Journal of

Function Spaces

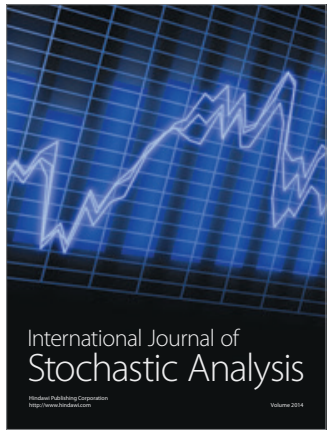

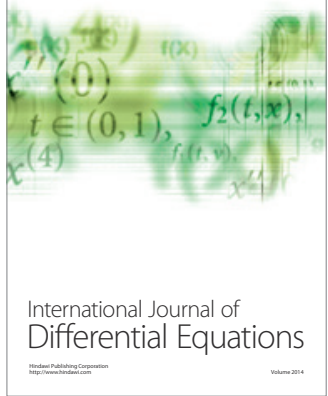
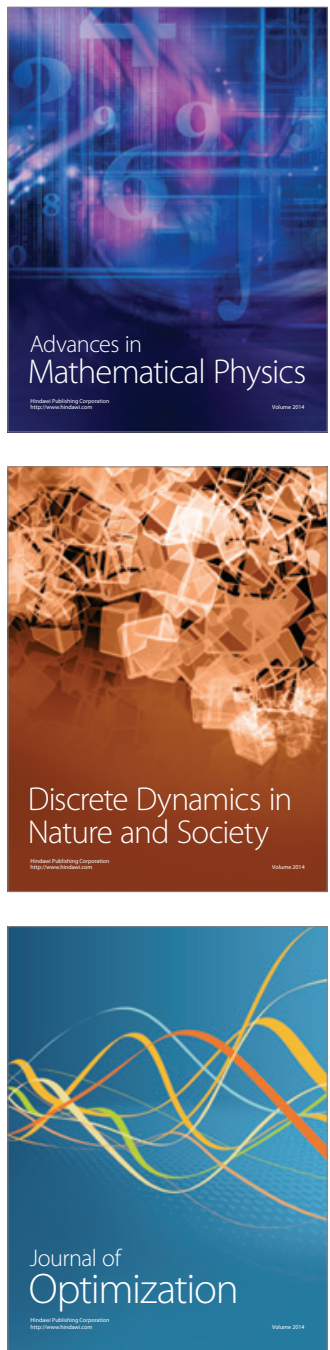